\title{
Guidelines on glaucoma and the demand for services
}

Dr Daniel Chalk (Research Fellow in Applied Healthcare Modelling and Analysis, University of Exeter Medical School, Exeter)

Michael Smith (Consultant Ophthalmologist, West of England Eye Unit, Royal Devon and Exeter Hospital)

Corresponding author: Dr Daniel Chalk, PenCHORD, Veysey Building, Salmon Pool Lane, Exeter, Devon, EX2 4SG

Telephone : 01392726081

Email :d.chalk@exeter.ac.uk 


\section{Abstract}

We produced a seasonalised forecast using a linear regression trendline to predict the level of demand for ophthalmology services at Royal Devon and Exeter hospital between 2013 and 2017. The model predicts an average annual increase in demand of $9.92 \%$, but a significant proportion of this predicted increased demand is for glaucoma services. Given the historical patterns of demand observed in the data, it is possible that the increases in demand for glaucoma services have arisen because of dramatically increased referral rates from community optometrists, following the publication by the National Institute for Clinical Excellence (NICE) of clinical guidelines on the diagnosis and management of glaucoma and ocular hypertension (NICE, 2009). As this patient cohort may be best served by assessment outwith secondary care, we would encourage other hospital managers and analysts to produce similar forecasts and consider alternative pathways of care for this patient group. 


\section{Introduction}

Glaucoma is a disease of the optic nerve which can lead to blindness if untreated and, in the UK, is the second most common cause of visual impairment (Bunce and Wormald, 2006). The management of glaucoma constitutes a significant proportion of the workload of eye departments, representing more than 1 million glaucoma related appointments in the NHS each year, including $23 \%$ of all Hospital Eye Service follow up appointments (Spry et al, 1999).

Although raised intraocular pressure (IOP) is considered the main risk factor for development of glaucoma (Sommer et al, 1991), many people have eye pressure outside of the normal range but never develop signs of glaucomatous damage to their optic nerves (Spry et al, 1999). This is known as ocular hypertension (OHT), and the number of individuals with OHT outnumber those with glaucoma (Spry et al, 1999).Traditionally, many patients with OHT were monitored by community optometrists as part of their routine eye exam (Spry et al, 1999), with referral to the Hospital Eye Service (HES) only occurring when there were signs of glaucoma, or the patient's IOP was found to be very high. Following the publication of NICE guidelines on Glaucoma in 2009 (NICE, 2009), several influential optometric industry bodies advised optometrists to refer all patients with IOP above $21 \mathrm{mmHg}$, which has been heavily criticised in an editorial by the Chair of the group who developed the NICE guidelines (Sparrow, 2013). The editorial describes the "unnecessary flooding of NHS glaucoma services with false positive referrals", which reflects the experience of many NHS eye units. For example, the number of new patients referred to the glaucoma screening clinic at the West of England Eye Unit increased from approximately 50 per month at the time of publication of the guidelines, to between 100 and 250 patients per month less than one year later (Figure 1). 


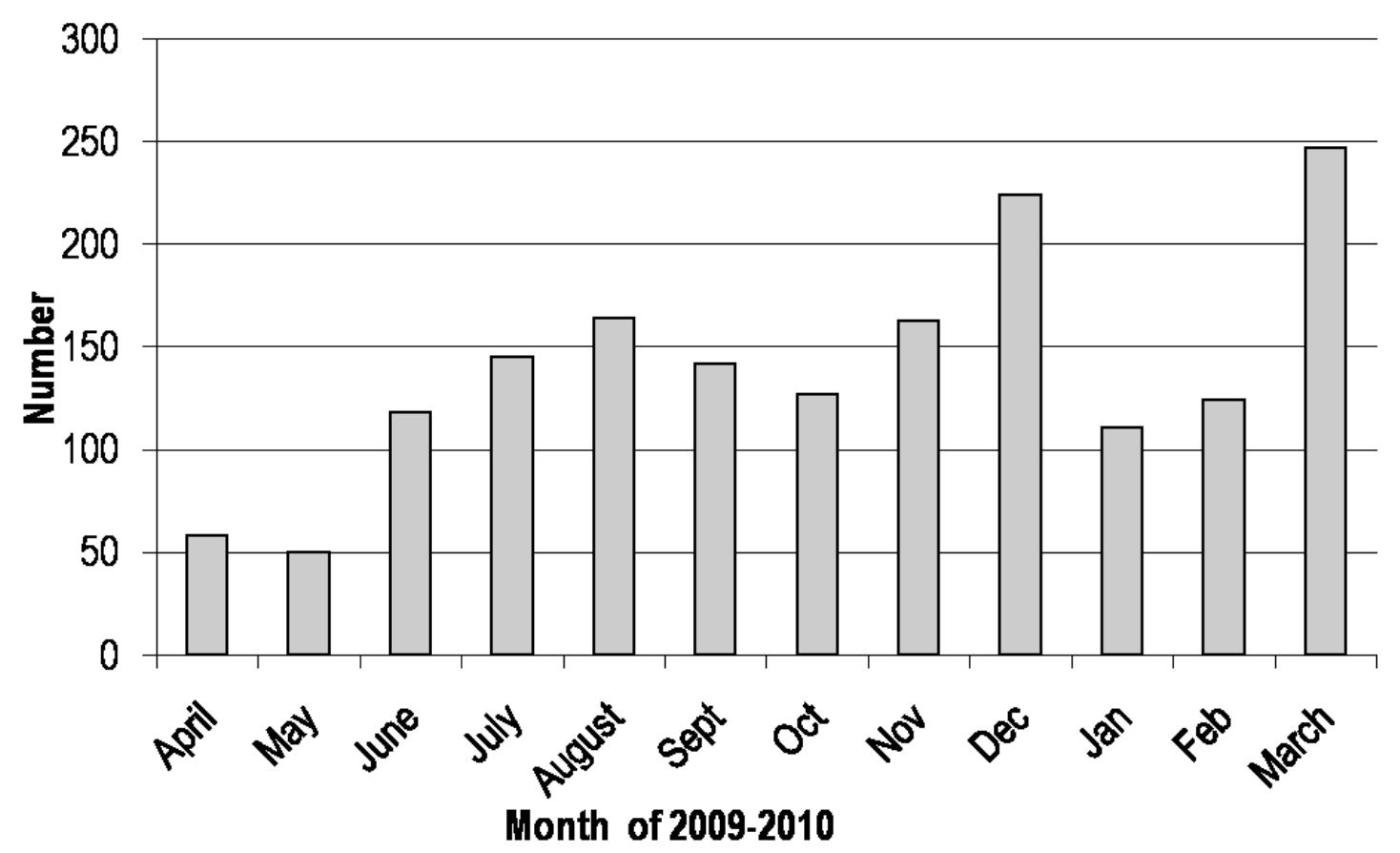

Figure 1. Number of new patients referred to WEEU Glaucoma Screening Clinic per month in 12 months following introduction of NICE Clinical Guideline 85 in April 2009.

Following the sharp increase in referrals in 2009-2010, attempts were made by the Royal College of Ophthalmologists and College of Optometrists to clarify referral guidelines, but these have not been considered to be successful, and referral rates remain considerably higher than pre-2009 (Sparrow, 2013).

In this study, we obtained historical data from Royal Devon and Exeter Hospital to produce a forecast of demand across ophthalmology services for the next five years. Specifically, we used a linear regression trendline to produce a seasonalised forecast, and determined the sub-specialties whose patterns of demand are predicted to have the largest impact on future levels of demand. A trendline uses regression analysis to provide a straight line of best-fit between time and demand in the historical data, and allows predictions of the future demand by extending this trendline. Linear regression is a common technique for predicting future demand patterns (Côté et al, 2001; Demir et 
al, 2012). A seasonalised forecast additionally takes into account any seasonal variations that may be inherent in a service's demand pattern (Côté et al, 2001), which has been shown to be present in healthcare services generally (Upshur, 2005) and which may be present in ophthalmology services (Iguchi et al, 2006; Leffler et al, 2010). We also broadly followed the processes and recommendations outlined by Finarelli and Johnson (2004).

\section{Methods}

We obtained anonymised historical data from Royal Devon and Exeter Hospital, comprised of 221,285 ophthalmology outpatient appointments between April 2007 and July 2012 for 61,781 patients. $10,616(4.8 \%)$ of the appointments were future appointments, and were therefore excluded from the analysis of the historical data.

Royal Devon and Exeter Hospital informed us that there was a long-standing historical appointment backlog across ophthalmology services, and that significant resources had been put in place in 2012 to reduce the backlog. Consequently, a section of the historical data may not be representative of the pattern of demand, because more patients than usual would have been seen during this period. We were supplied with overdue appointment data from September 2009 to September 2012, which represented those appointments for which a patient had been invited to a follow-up appointment later than agreed, and we plotted this data graphically (Figure 2). We observed that the backlog remained relatively stable up until May 2012, at which point significant reductions could be seen. Therefore we excluded historical data from May 2012 from our analysis. We also excluded 2007 data from the forecasting process as we only had data for part of the year, and appointment records for which we did not know the sub-specialty to which the appointment belonged. This left us with 122,591 appointments. 


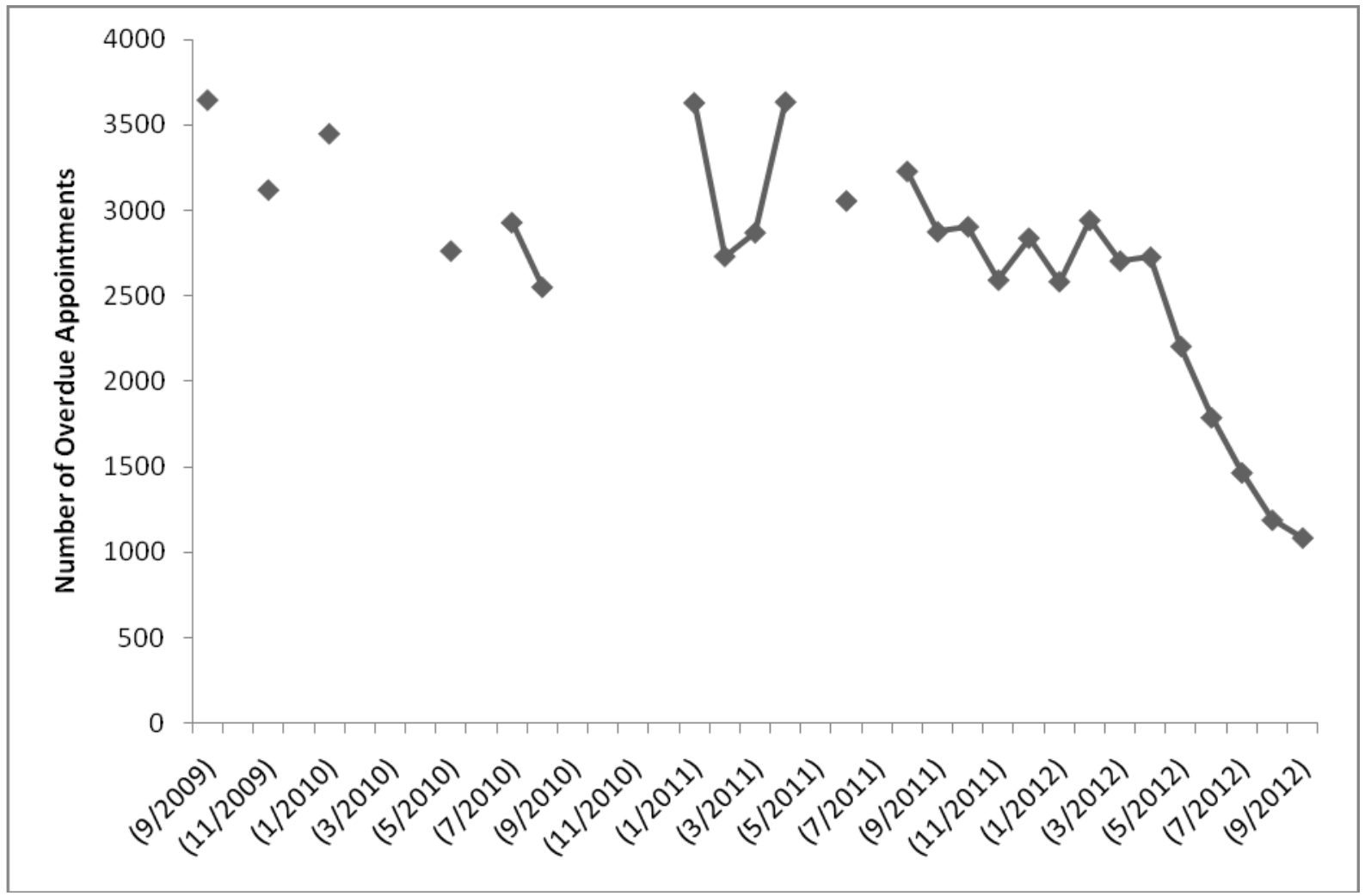

Figure 2. The number of overdue appointments across Royal Devon and Exeter Ophthalmology

Services between September 2009 and September 2012.

Finarelli and Johnson (2004) recommend that historical data is checked for any unusual or unexpected trends, in order to assess the integrity of the data set. We looked at three ratios that we would expect to remain relatively constant over time - (1) the ratio of appointment attendances to referrals for surgery, (2) the ratio of appointment attendances to referrals for a follow-up appointment, and (3) the ratio of appointment attendances to discharges from the clinic. We excluded 2012 appointments from this analysis as we had only partial usable data for this year. We found that although there was some reduction in the ratio of attendance to follow-up appointment referral, both of the other ratios remained extremely stable over time (Figure 3), which offers confidence in the integrity of the data. 


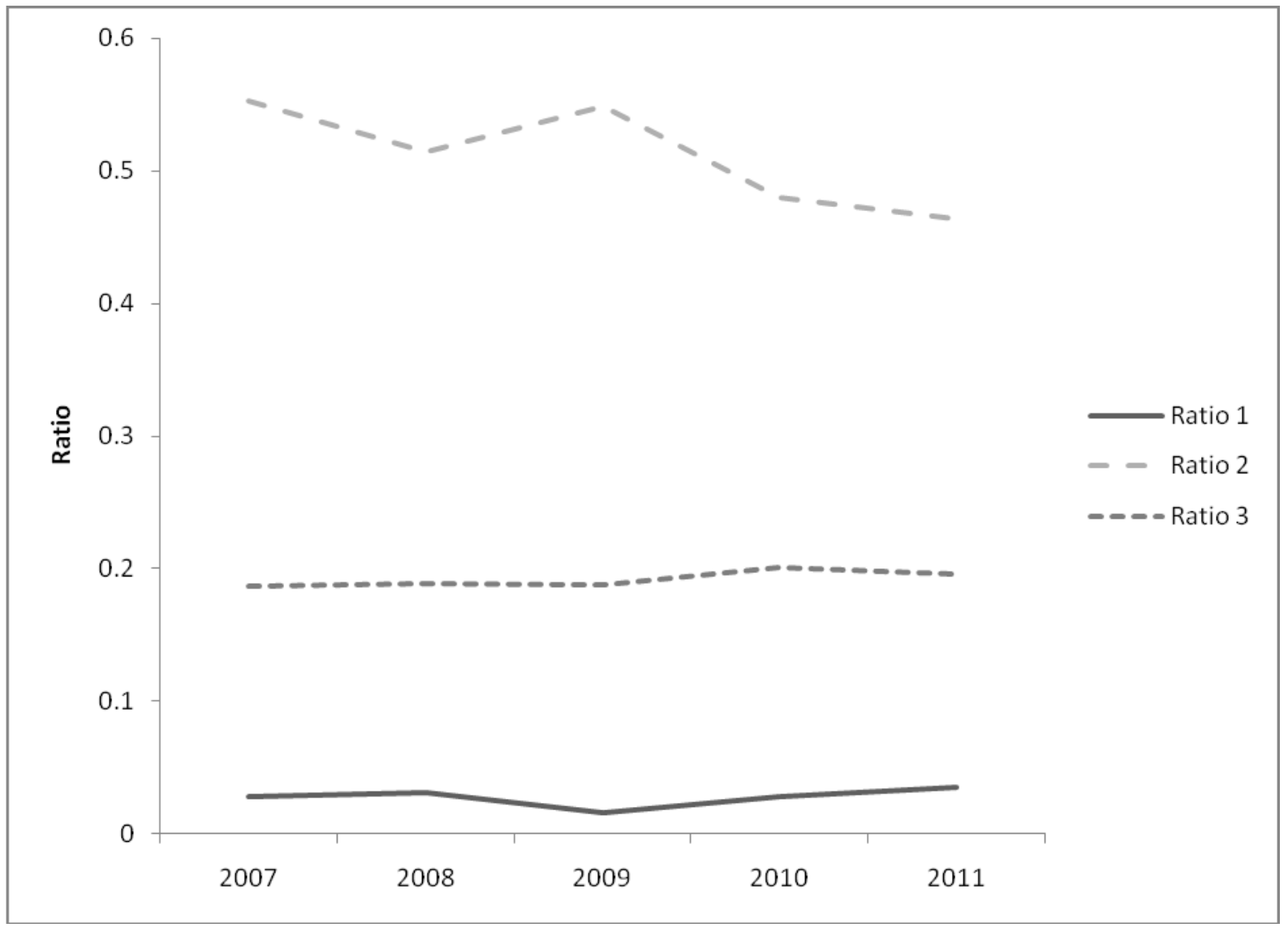

Figure 3. The stability of tested ratios in the historical data between 2007 and 2011.

In order to produce a seasonalised forecast using a linear regression trendline, we followed the method of Côté et al (2001). First, we identified the period of representative historical demand to analyse. Second, for each ophthalmology sub-specialty, we calculated the average monthly demand over this period along with the average demand for each calendar month. This allows us, for each sub-specialty and for each calendar month, to calculate a seasonal index by dividing the average demand for that calendar month by the average monthly demand. Third, we remove the seasonal effects from the data by dividing the demand for each month and year by the relevant seasonal index. Finally, we use least squares regression on the converted demand data to produce a linear trendline, from which we can derive predictions of future demand.

Before producing the forecast of future ophthalmology demand, we attempted to use the model to predict the current level of demand, in order to assess the model's ability to produce a reliable 
demand forecast. We supplied the model with data from 2008 to 2010 (inclusive), and assessed its ability to predict the level of ophthalmology demand in 2011. Figure 4 compares the actual level of demand in 2011 for three core ophthalmology sub-specialties at Royal Devon and Exeter Hospital which were those identified by the hospital as being the most susceptible to increased demand with the predictions of the model. We found that the model was able to predict the level of ophthalmology demand in 2011 relatively well, particularly for the diabetic and sub-specialty.

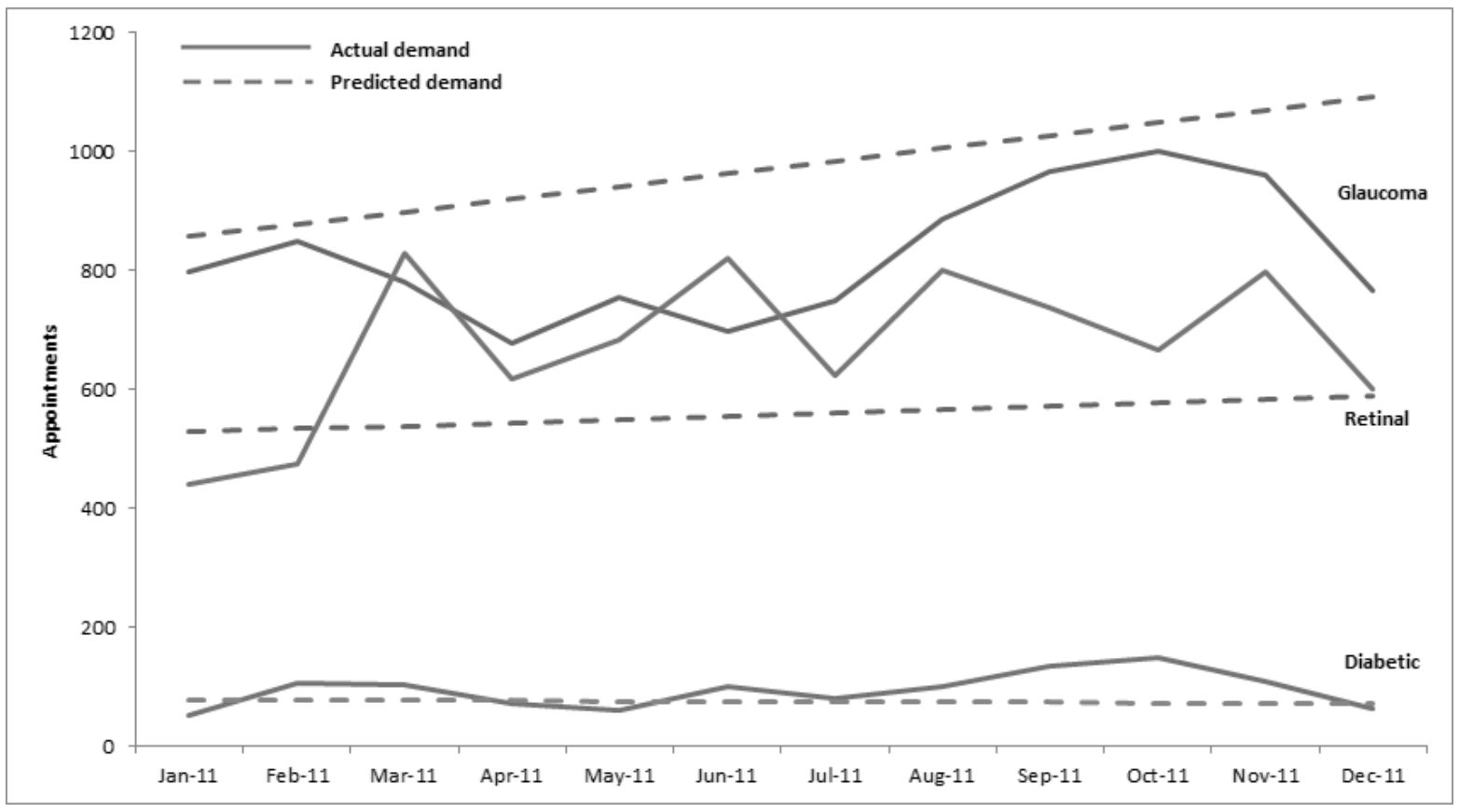

Figure 4. Comparison of actual demand in 2011 and predicted demand for 2011 from the model, for three core ophthalmology sub-specialties.

\section{Results}

The forecasting model predicts an increase in the overall level of ophthalmology demand between 2012 and 2017. Specifically, the model predicts there will be an average annual increase in demand of 9.92\% across ophthalmology services. A detailed breakdown of the predicted level of demand for each year and sub-specialty is shown in Table 1. Figure 5 shows the predicted demand for the three core ophthalmology sub-specialties. 


\begin{tabular}{|c|c|c|c|c|c|c|c|}
\hline & 2011 & 2012 & 2013 & 2014 & 2015 & 2016 & 2017 \\
\hline A\&E & 2891.439 & 3609.573 & 4326.399 & 5042.897 & 5759.395 & 6477.529 & 7194.355 \\
\hline Diabetic & 897.8562 & 806.0823 & 714.4756 & 622.9107 & 531.3458 & 439.5718 & 347.9651 \\
\hline Dystonia & 382.981 & 433.75 & 484.4264 & 535.0797 & 585.733 & 636.502 & 687.1784 \\
\hline General & 9732.538 & 10060.86 & 10388.59 & 10716.17 & 11043.74 & 11372.07 & 11699.8 \\
\hline Glaucoma & 11699.117 & 14785.639 & 17866.535 & 20946.028 & 24025.511 & 27112.033 & 30192.93 \\
\hline Laser & 1346.697 & 1391.811 & 1436.844 & 1481.855 & 1526.867 & 1571.981 & 1617.014 \\
\hline Retinal & 6701.578 & 7511.484 & 8319.913 & 9127.974 & 9936.035 & 10745.94 & 11554.367 \\
\hline Neonatal & 63.66899 & 51.3096 & 38.97273 & 26.6415 & 14.31026 & 1.950873 & -10.386 \\
\hline Ocular & 2226.0684 & 2488.3464 & 2750.1468 & 3011.8275 & 3273.5083 & 3535.7873 & 3797.5877 \\
\hline $\begin{array}{l}\text { Paediatric } \\
\text { Ophthalmology }\end{array}$ & 1693.218 & 1725.899 & 1758.521 & 1791.127 & 1823.734 & 1856.415 & 1889.036 \\
\hline Private & 384.7037 & 404.0983 & 423.4575 & 442.8079 & 462.1583 & 481.5528 & 500.9121 \\
\hline Thyroid & 73.7043 & 69.5473 & 65.39787 & 61.25033 & 57.1028 & 52.9458 & 48.79637 \\
\hline
\end{tabular}

Table 1. Predicted level of demand (appointments) per year for each sub-specialty.

The model forecasts a significant increase in the level of demand for glaucoma services, with a predicted increase of 3,081 appointments per year. This represents $58.8 \%$ of the predicted annual increase across ophthalmology services at Royal Devon and Exeter Hospital. The historical data indicates that the number of appointments for glaucoma services increased dramatically in 2009, increasing from 1,713 appointments in 2008 to 6,292 appointments in 2009 - a 367\% increase, compared to a 152\% increase of 584 appointments between 2007 and 2008. 


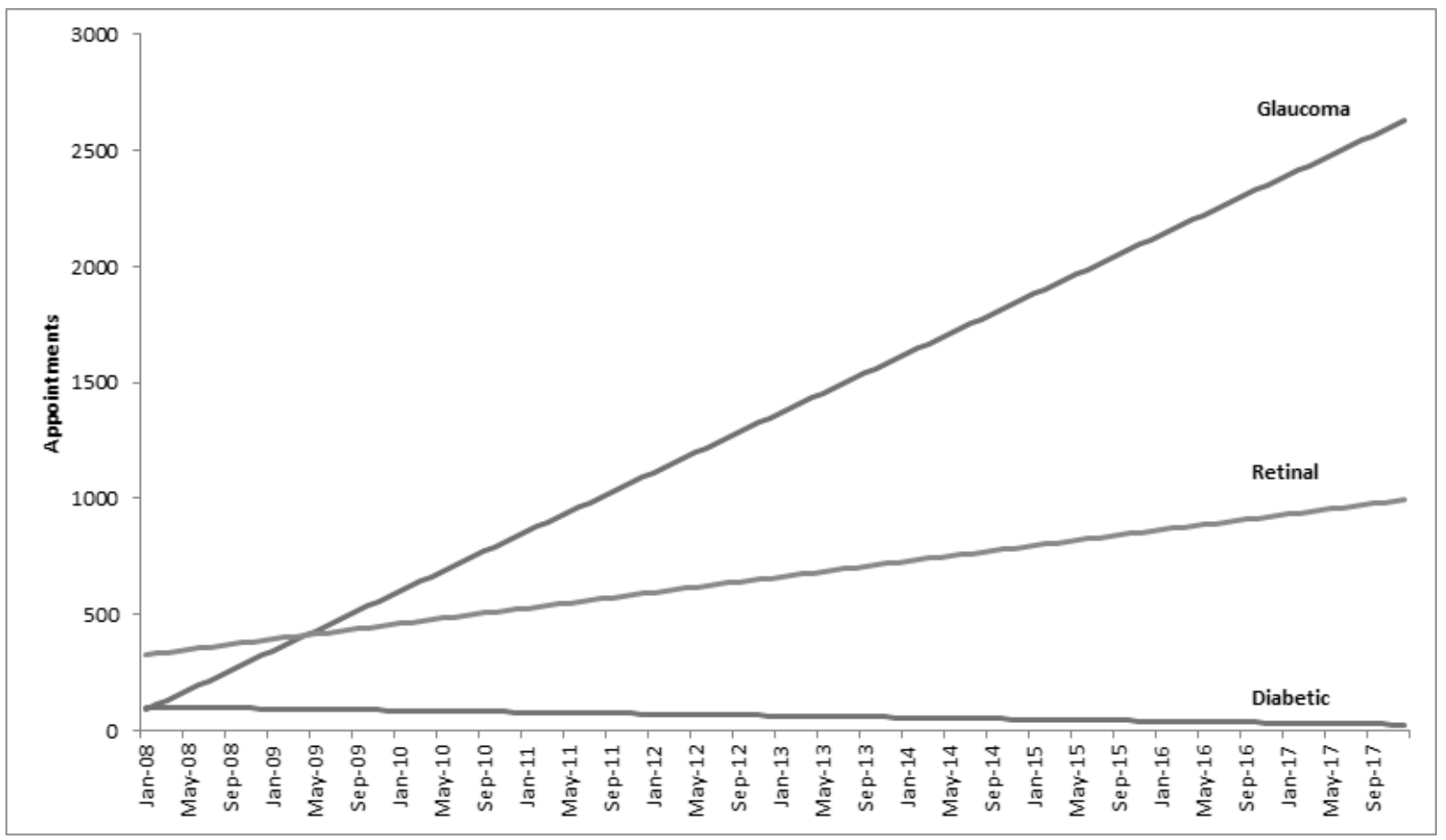

Figure 5. Predicted level of demand (appointments) for the threeophthalmnology sub-specialties at Royal Devon and Exeter Hospital.

Large increases in demand are also predicted for the Retinal and Accident and Emergency subspecialties, with forecast increases of 808 appointments per year and 717 appointments per year, respectively. Conversely, the trendlines predict a minor decrease in demand for the diabetic subspecialty, with average annual decreases of 92 appointments.

\section{Discussion}

We developed a seasonalised trendline model using data supplied by Royal Devon and Exeter Hospital. Our model predicts that, on average, there will be a non-trivial increase in demand for ophthalmology services over the next five years at Royal Devon and Exeter Hospital. Furthermore, the majority of this increased demand is for glaucoma services.

Our analysis of the historical data supplied by the hospital indicated that there was a significant increase in the number of glaucoma-related appointments between 2008 and 2009. When we discussed this with ophthalmology managers and analysts at the hospital, they advised us of the 
change in referral behaviour for glaucoma following the publication of the Glaucoma NICE guidelines in 2009 (NICE, 2009). It would therefore seem that the majority of the forecasted additional demand for ophthalmology services may have arisen because of the increased number of referrals resulting from the interpretation of these guidelines by community optometrists.

To help determine the likelihood of this hypothesis, we ran our forecasting model again, but only using data for 2007 and 2008 to generate the trendlines, which represents the period of time before the guidelines were introduced. We found that the predicted average annual increase in demand for glaucoma services reduced significantly, from $15.39 \%$ per year to $8.11 \%$ per year. This would further support the notion that the introduction of the guidelines has led to increasing demand for glaucoma-related services. It is worth noting that, using this shorter data set, the predicted increases in demand across ophthalmology services actually increased, albeit very slightly, from 9.92\% to $10.24 \%$ (or from 5,236 appointments per year to 6,040 appointments per year, in absolute terms). However, this is likely the result of the smaller sample set used to generate the trendline, which would inherently place more emphasis on atypical variations in demand.

It is somewhat surprising that our model predicts a small decrease in demand for diabetic subspecialty ophthalmology appointments, particularly given the significant increases in prevalence rates of diabetes (Shaw, Sicree and Zimmet, 2010), giving rise to concerns that diabetic eye services do not have the resources to accommodate this increasing demand (Chalk et al, 2012). In reality, the forecasted decrease here is very minor, and could be considered as a flatlining of the demand. Given that demand is defined here as the number of appointments provided to patients, this flatlining may indicate that the diabetic eye services at Royal Devon and Exeter Hospital are already at saturation point, which would correspond with a previous paper, in which we proposed a way in which diabetic eye services such as those at Royal Devon and Exeter Hospital could accommodate an increasing prevalence of diabetes (Chalk et al, 2012). 
Our forecasting model has brought to light the influence of demand for glaucoma-related services on potentially significant increases in demand for ophthalmology services more generally. We would therefore recommend that other healthcare managers produce similar forecasts to determine whether increased referral rates following the introduction of the NICE guidelines in 2009 may be leading to increased demand for their ophthalmology services. Furthermore, as many of these cases are false positive referrals which may be diverting resources from patients with vision threatening glaucoma (Sparrow, 2013), and attempts by the Royal College of Ophthalmologists and College of Optometrists to nationally reduce unnecessary referrals by clarifying referral guidelines do not seem to have been successful (Sparrow, 2013), local solutions may need to be investigated.

\section{Acknowledgements}

We would like to thank Richard Blackwell, Abbie Sowden, Philip Luke, Daniel Byles, Rebecca Snell, Lynsey Lowthian and Em Wilkinson-Brice of Royal Devon and Exeter NHS Foundation Trust, and Adam Carrick and Hannah Tappof Devon PCT for providing feedback, advice and guidance throughout the project.

\section{Key Points}

- We produced a seasonalised trendline-based forecast of ophthalmology demand over the next five years at Royal Devon and Exeter Hospital

- We predict an average annual increase in demand for ophthalmology services of $9.92 \%$

- The majority of additional forecasted demand is predicted to come from glaucoma services

- Significant increases in glaucoma demand seem to have arisen because of increased referral rates following NICE guidelines on glaucoma in 2009

\section{Literature Cited}


Bunce C, Wormald R (2006) Leading causes of certification for blindness and partial sight in England \& Wales. BMC Public Health 6:58-64

Chalk D, Pitt M, Vaidya B, Stein K (2012) Can the retinal screening interval be safely increased to 2 years for type 2 diabetic patients without retinopathy? Diabetes Care 35(8): 1663-1668

Côté M J, Tucker S L (2001) Four methodologies to improve healthcare demand forecasting. Healthcare Financial Management 55(5): 54-58

Demir E, Chahed S, Chaussalet T, Toffa S, Fouladinajed F (2012) A decision support tool for health service re-design. J Med Syst 36(2): 621-630

Finarelli H J, Johnson T (2004) Effective demand forecasting in 9 steps. Healthcare Financial Management 58(11): 52-58

Iguchi Y, Ito Y, Kikuchi M et al (2006) Seasonal variations of acute massive submacular haemorrhage associated with age-related macular degeneration. $\mathrm{Br} \mathrm{J}$ Ophthalmol 90: 1256-1258

Leffler C T, Davenport B, Chan D (2010) Frequency and seasonal variation of ophthalmology-related internet searches. Can J Ophthalmol 45(3): 274-279

Moseley M J (1995) Non-contact tonometry. Ophthalmic and Physiological Optics 15(S2): S35-S37

Myint J, Edgar D F, Kotecha A, Murdoch I E, Lawrenson J G (2011) A national survey of diagnostic tests reported by UK community optometrists for the detection of chronic open angle glaucoma. Ophthalmic and Phsyiological Optics 31(4): 353-359

National Institute for Health and Clinical Excellence (NICE) (2009) Glaucoma: diagnosis and management of chronic open angle glaucoma and ocular hypertension. http://guidance.nice.org.uk/CG85, accessed 1 June 2013 
Shaw J E, Sicree R A, Zimmet P Z (2010) Global estimates of the prevalence of diabetes for 2010 and 2030. Diabetes Res ClinPract 87(1): 4-14

Sommer A, Tielsch J M, Katz J et al (1991) Relationship between intraocular pressure and primary open angle glaucoma among white and black Americans. Arch Ophthalmol 109:1090-1095

Sparrow JM (2013) How nice is NICE?. Br J Ophthalmol97:116-117

Spry PG, Spencer IC, Sparrow JM, Peters TJ, Brookes ST, Gray S, et al (1999) The Bristol Shared Care Glaucoma Study: reliability of community optometric and hospital eye service test measures. $\mathrm{Br} \mathrm{J}$ Ophthalmol83:707-12.

Upshur R (2005) To everything there is a season: hospitalizations in Ontario demonstrate strong evidence of seasonality and predictability. Health Quarterly 8(4): 24-26 